\title{
Evaluation du potentiel ethnobotanique des populations rurales au Sud et au centre du Bénin
}

\author{
J. DJEGO, S. DJEGO-DJOSSOU*, Y. CAKPO, P. AGNANI et B. SINSIN \\ Laboratoire d'Ecologie Appliquée, Faculté des Sciences Agronomiques, Université d'Abomey-Calavi, 01 BP \\ 526 Cotonou, Bénin. \\ *Corresponding author, E-mail: gdjego@yahoo.fr
}

\section{RESUME}

Les ressources végétales constituant pour les ruraux, d'importantes sources de soins médicaux et d'aliments, sont mal gérées du fait de la forte emprise humaine. Cette étude diagnostique, évalue les connaissances ethnobotaniques des populations rurales au Bénin, en vue d'analyser le niveau de conservation par rapport aux anciennes collections des années 1935 et 1940 effectuées par Laffite. La méthode de collecte de données est faite d'enquêtes ethnobotaniques et de relevés floristiques. Au total, 232 espèces utiles ont été collectées dont 25,86\% recensées par Laffite. Parmi elles, 96\% sont notamment utilisées en médecine traditionnelle, $20 \%$ en spiritualité, $13 \%$ dans l'alimentation et $3 \%$ en technologie. Certaines espèces mentionnées par Laffite ont disparu (Milletia thonningii, Premna hispida, Bryophyllum pinnatum, Spilanthes uliginosa, Caesalpinia bonduc, Tephrosia vogelii et Cissampelos mucronata) des terroirs, tandis que d'autres sont vulnérables en déclin (Afraegle paniculata, Antiaris toxicaria, Carissa edulis, Commiphora africana, Crateva adansonii, Pseudocedrela kotschyi, Securidaca longipedunculata, Leptadenia hastata et Tylophora camerunica). L'urbanisation, l'agriculture itinérante, les changements climatiques et l'introduction d'espèces exotiques sont autant de facteurs expliquant cette vulnérabilité. La prise de mesure de protection, l'installation de jardins de case et l'adoption d'approche de gestion durable de cette phytodiversité sont urgentes.

(c) 2011 International Formulae Group. All rights reserved.

Mots clés : Ethnobotanique, Laffite, phytodiversité, vulnérabilité, conservation, Bénin.

\section{INTRODUCTION}

Les formations forestières fournissent de très nombreuses ressources animales et végétales qui sont des sources d'alimentation, de plantes médicinales, de fourniture en boisénergie, et bois d'œuvre pour les populations locales (Sokpon et Lejoly, 1996 ; Ros-Tonen, 1999 ; Goussanou et al., 2011). Le milieu tropical auquel appartient l'Afrique en grande partie, dispose d'une diversité biologique très élevée, à tel point que l'avenir de notre planète dépend de sa survie. Mais cette survie se voit menacée par des attaques catastrophiques dues aux variations climatiques provoquées ou non et aux interventions humaines contrôlées ou non (Adjanohoun et al., 1999 ; Delvaux et al., 2010). La perte de couverture forestière a atteint dans la décennie 1990-2000, 14,2 millions ha/an et l'Afrique, avec seulement $16,8 \%$ du couvert mondial, a contribué pour 
$56 \%$ à cette réduction du couvert forestier (Djègo, 2006). C'est le cas en Afrique au Sud du Sahara et particulièrement au Bénin où les ressources génétiques, précisément les espèces médicinales s'amenuisent progressivement à cause de leur utilisation abusive.

Dans le monde, les couvertures forestières s'amenuisent dangereusement (Djègo and Oumorou, 2009) et très souvent, leur existence est remise en question (Yessoufou, 2005). Les menaces qui pèsent sur ces formations végétales comprennent les pratiques culturales, l'élevage, l'exploitation $\mathrm{du}$ bois et des Produits Forestiers Non Ligneux (Sokpon and Agbo, 2001 ; Sinsin et al., 2009), la croissance démographique et urbanisation (Yessoufou, 2005). L'Afrique de l'Ouest perd chaque année $4 \%$ de forêt dense (Harrison, 1991). Dans le cas particulier du Bénin, la couverture forestière est passée de 4 923000 ha en 1990 à 4625000 ha en 1995 soit une perte totale de 298000 ha de forêts en 5 ans (FAO, 1999). Le Bénin perd 60000 ha de forêt par an soit un taux annuel de déforestation évalué à $1,2 \%$. Cette déforestation n'est pas sans conséquences sur la conservation des ressources biologiques et notamment sur les plantes médicinales qui demeurent encore une source de soins médicaux dans les pays en voie de développement, en l'absence d'un système médical moderne (Tabuti et al., 2003 ; Deleke et al., 2009). En effet, la destruction des forêts tropicales est la cause essentielle de réduction de la diversité biologique (Djègo and Sinsin, 2006) et présente des conséquences économiques et écologiques graves. De nombreuses espèces forestières utiles sontelles vulnérables de nos jours ou menacées d'extinction? Quand n'est-il de la conservation de savoirs ethnobotaniques traditionnels? Une attention particulière devra être accordée à la conservation et au maintien de l'habitat, pour la sauvegarde des plantes médicinales en péril. En effet, depuis la récession économique touchant la plupart des pays africains sub-sahariens, un nouveau type de commerce a fait son apparition; celui d'inonder anarchiquement les marchés nationaux et internationaux, de plantes thérapeutiques. Pour qu'il y ait disponibilité durable de ces plantes médicinales, il est important de penser dès à présent à leur conservation. D'où l'intérêt de la présente étude effectuée dans neuf (09) communes au Sud et au Centre du Bénin qui a pour objectif d'évaluer les connaissances ethnobotaniques des populations rurales et la flore utile en vue d'analyser le niveau de conservation par rapport aux anciennes collections des années 1935 et 1940 effectuées par Laffite. Il s'agira entre autre d'identifier les espèces menacées ainsi que les différentes stratégies de leur conservation.

\section{MATERIEL ET METHODES Milieu d'étude}

La présente étude a été effectuée dans les départements du Littoral (Commune de Cotonou), de l'Atlantique (Communes d'Allada et de Ouidah), du Zou (Communes d'Abomey et de Djidja), des Collines (Communes de Dassa-Zoumè, de Glazoué, de Savalou et de Bantè). Le Tableau 1 présente les caractéristiques démographiques, climatiques et édaphiques de ces sites de la zone d'étude (Figure 1).

\section{Méthodologie}

La principale source de données exploitée pour ce travail relève des enquêtes sur l'exploitation des ressources végétales dans les différentes localités ainsi que les impacts des activités agricoles sur ces espèces. Ces données ont été collectées en utilisant la technique d'entretiens structurés basés sur des questionnaires permettant aux enquêteurs de mieux orienter leur discussion avec les enquêtés. Les informations recueillies concernent le profil de chaque enquêté (âge, niveau d'études, situation familiale, revenu et lieu de résidence); la plante (nom local commun, usages, partie utilisée, mode de préparation, période de collecte, type de plante et prix). Toutes les espèces ont été mentionnées par les enquêtés par leur nom 
commun. L'identification taxonomique des espèces a été réalisée sur place à l'aide de flores ou ultérieurement à l'herbier national du Bénin. A cet effet, des herbiers ont été réalisés.

Des techniques d'analyse descriptive simple ont été effectuées. La méthode Tramyl IV a permis de dégager les espèces les plus significatives pour traiter chaque maladie.

Les données inscrites sur des fiches de données brutes ont été transférées dans une base de données et traitées par le logiciel de traitement statistique SPSS et STATISTICA.

\section{RESULTATS}

\section{Profil des enquêtés}

Six groupes ethniques ont été rencontrés au cours de l'enquête. Il s'agit des Aïzo (commune d'Allada) ; Fons (communes d'Abomey, de Cotonou, de Ouidah et de Djidja), Idaasha (Commune de DassaZoumé), Ifè (Commune de Savalou), Isha (Commune de Bantè); Mahi (Communes de Dassa-Zoumé et de Savalou).

Dans le Sud et au Centre du Bénin, l'âge moyen des enquêtés tourne autour de 65 ans. Le ratio Homme/Femme enquêté est largement favorable pour le sexe masculin 94\% d'hommes pour $6 \%$ de femmes dans le Centre tandis qu'il est de $54 \%$ pour le sexe féminin et de $46 \%$ pour les hommes dans le Sud. Cette tendance à la féminisation de l'échantillon au sud du Bénin est due au fait que dans la commune de Cotonou, seules les vendeuses de plantes médicinales ont été questionnées.

Dans la plupart des cas, les enquêtés mènent des activités doubles (AgriculteurPhytothérapeute) ou multiples. En se fondant sur les activités prioritaires, au Centre du Bénin, 61,4\% des enquêtés sont des agriculteurs (Figure 2), 25,5\% des phytothérapeutes ou guérisseurs traditionnels, $3,4 \%$ sont des ménagères et $9,7 \%$ exercent des métiers qui sont plus ou moins liés aux plantes (maçon, forgeron, artiste, apiculteur, médecin vétérinaire etc.).
Dans le Sud, l'échantillon est constitué de $50 \%$ de vendeuses de plantes médicinales, $25 \%$ de tradipraticiens, $11 \%$ d'agriculteurs et enfin $14 \%$ sont des tailleurs, coiffeuses et autres.

\section{Inventaire et exploitation des ressources}

Dans la zone d'étude 232 espèces utiles ont été recensées dont 190 au Sud et 42 au Centre. Parmi ces espèces s'y retrouve la majorité des espèces collectées par Laffite entre les années 1935 et 1940. Ainsi, au Sud et au Centre Bénin, respectivement 15,8\% et $71,4 \%$ des espèces collectées ont été recensées par Laffite.

La fréquence d'utilisation des plantes médicinales selon les localités investiguées présente une différence significative au Sud $(\mathrm{p}$ $<5 \%)$ et non significative au Centre ( $p>5 \%)$ du Bénin. Ainsi, l'analyse de la répartition des espèces par communes (Figure 3) révèle qu'au Sud, le grand nombre d'espèces est observé à Abomey (104 espèces) et à Ouidah (98 espèces) tandis qu'à Allada et Cotonou, s'observent les plus faibles nombres (respectivement 76 et 73 espèces). Toutefois, les espèces mentionnées par Laffite sont présentes dans toutes les communes en effectifs presque semblables (Figure 3). Ceci s'explique par le fait que les espèces de Laffite sont presque toutes médicinales et reconnues par les populations. A Abomey (ville historique) et Ouidah (ancienne ville portuaire), le grand nombre d'espèces recensées signale l'attachement des populations aux connaissances traditionnelles liées à l'usage des plantes utiles; tandis qu'à Cotonou et Allada, le caractère cosmopolite de ces villes influence négativement le maintien et la transmission des connaissances ethnobotaniques.

\section{Représentations socio-anthropiques des végétaux et leurs usages}

Les connaissances ethnobotaniques des populations du Sud et du Centre Bénin sont traditionnellement riches à cause de la diversité des groupes ethniques, des coutumes 
et des traditions mais aussi de la diversité des espèces végétales et de leur habitat. La flore joue un rôle important dans la vie des populations rurales. Elle est utilisée à plusieurs fins. Dans l'ordre d'importance, on distingue (Figure 4) les plantes utilisées en médecine traditionnelle (73\%), en spiritualité : sorcellerie, mauvais sorts, conflits $(15 \%)$, en alimentation (10\%) et en technologique (2\%). Il ressort que les populations rurales de la zone d'étude sont fortement attachées aux ressources naturelles végétales qui constituent leur principale source d'approvisionnement en médicaments, en aliments, et d'un tas d'utilisations importantes de la vie quotidienne.

\section{Fréquence d'utilisation des plantes utiles dans la zone d'étude}

Sur les 232 espèces recensées au Sud et au Centre du pays, 178 sont spontanées, récoltées dans les formations végétales; 24 espèces sont importées d'autres régions et une trentaine cultivées dans les jardins de case. Au sein des populations, $82,8 \%$ des enquêtés utilisent les espèces végétales médicinales prélevées dans les formations végétales (Figure 5), 12,1\% exploitent les espèces cultivées autour des habitations et $5,2 \%$ utilisent aussi des espèces issues d'autres régions. Cette faible utilisation d'espèces importées se justifie par leur prix élevé.

Parmi les espèces utilisées en médecine traditionnelle, Carissa edulis, Clausena anisata, Crateva adansonii, Anogeissus leiocarpa et Pavetta crassipes, sont les plus exploitées. En spiritualité, les espèces fréquemment utilisées sont: Commiphora africana, Clitoria ternatea, Vernonia amygdalina, Vernonia cinerea et Philenoptera cyanescens. En alimentation, il s'agit de Boerhavia diffusa, Pterocarpus santalinoides et Vernonia amygdalina. Antiaris toxicaria bien qu'ayant des usages spirituel et médicinal est également utilisée dans la fabrication des pirogues. Les populations font recours au Leptadenia hastata comme cure dent.
$\mathrm{Au}$ centre du Bénin, une quinzaine d'espèces sont déclarées alimentaires. Elles sont utilisées soit pour la cause alimentaire sensus stricto (Vernonia spp, Prosopis africana, Carissa edulis, Grewia mollis etc.), soit pour le traitement des maladies (Afraegle paniculata, Euphorbia convolvuloides, Vernonia cinerea, etc.). En fonction des types de maladies, ce sont les espèces qui ont des vertus contre le paludisme, le traitement des maladies infantiles et les aphrodisiaques qui sont les plus utilisées. Les organes utilisés sont identiques à celles énumérées pour Laffite. En dehors des espèces de Laffite, il a été recensé d'autres espèces dont les plus fréquemment utilisées en médecine traditionnelle sont: Caesalpinia bonduc, Ocimum gratissimum, Uvaria chamea, Phyllanthus amarus, Mangifera indica, etc.

\section{Drogues utilisées, espèces significatives et usages}

Les populations de la zone d'étude ont signalé huit drogues végétales (Figure 6) qui entrent dans les préparations de recettes thérapeutiques. Il s'agit de: feuille, racine, écorce, fruit, fleur, sève, latex et bulbe. Parmi ces drogues, les plus utilisées sont les feuilles (41\%), les racines $(20 \%)$ et les écorces $(15 \%)$. Le latex et le bulbe étant les organes ayant les plus faibles fréquences de citation (2\%). Parfois, la drogue végétale peut être constituée par la plante entière. C'est le cas de Euphorbia convolvuloides et de Vernonia cinerea.

Le Tableau 2 indique l'utilité de quelques plantes significatives ainsi que les drogues et le nombre de pathologies indiqués par les populations au Sud et au Centre du Bénin.

La majorité de ces espèces sont utilisées dans un nombre important de maladies (paludisme, maladies infantiles, infections virales et bactériennes, affections cardio-vasculaires, dermatoses, troubles gynécologiques, etc.). Elles sont souvent utilisées comme aphrodisiaque (Carissa edulis) ou contre le paludisme, soit après 
accouchement et pour les nourrissons (Caesalpinia bonduc). Leurs racines et écorces sont prélevées en abondance ou utilisées majoritairement par les tradipraticiens dans les cérémonies de lutte contre la sorcellerie. Ce sont des espèces ayant des vertus spirituelles (chance, conflits). Mais elles ne sont cultivées pour assurer la disponibilité de la ressource. Chez Caesalpinia bonduc par exemple, non seulement l'espèce est sollicitée à des fins thérapeutiques, ces graines sont aussi utilisées pour le jeu de «Domino »; ce qui constitue une réelle menace pour la pérennité de l'espèce. Une autre menace évoquée pèse sur Carissa edulis; du fait de l'exploitation intense de ses racines et de sa régénération difficile due à la toxicité de ses semences.

\section{Prélèvement des organes et disponibilité des espèces}

Selon les enquêtés, les prélèvements se font en fonction des besoins et ceci au détriment de la survie des espèces. Il est recommandé d'effectuer les prélèvements tôt le matin, car ils estiment que les vœux formulés au réveil avant de manger du sel ou d'échanger des paroles avec quelqu'un sont très souvent exhaussés. Toutefois, dans des cas spécifiques des maladies complexes à connotation de sorcellerie ou dans des cas d'envoûtement ou de désenvoûtement, il est nécessaire de prendre des dispositions particulières pour prélever le matériel végétal. Ainsi, avant de prélever des feuilles, des tiges feuillées ou des écorces de Detarium senegalense ou le Detarium microcarpum pour un désenvoûtement ou pour la protection contre la sorcellerie, il faudra prendre les dispositions suivantes :

- Etre dans un état spirituel pur (éviter un acte sexuel $24 \mathrm{~h}$ avant) ou bien en cas d'urgence prendre un bain de purification avec Ocimum canum, Ocimum basilicum, Portulaca oleracea et Newbouldia laevis avant d'aller vers le végétal.
- Prélever les écorces du côté Est et du côté Ouest pour certains, dans les quatre points cardinaux pour d'autres.

- Remercier le végétal et partir sans regarder derrière pour assurer la réussite du rituel.

L'aspect métaphysique apparaît dominant dans les prélèvements des plantes. Le caractère scientifique est absolument absent des dispositions particulières de prélèvement des espèces. Enfin, il faudra obtenir des autorisations spéciales des gardiens des sites sacrés lorsque l'on veut prélever des espèces comme Commiphora africana.

En ce qui concerne la disponibilité des ressources, plusieurs espèces végétales recensées sont encore répandues dans le milieu mais d'autres sont menacées. Une comparaison faite avec la liste des espèces recensées par Laffite au cours des années 1935 et 1940, a permis de se rendre compte de la vulnérabilité de nos jours de certaines d'entre elles. Ainsi, se distinguent :

- Des plantes disparues dans la nature et qui n'existent plus qu'en culture ; il s'agit de Milletia thonningii, Premna hispida, Bryophyllum pinnatum, Spilanthes uliginosa, Caesalpinia bonduc, Tephrosia vogelii et Cissampelos mucronata.

- Des plantes vulnérables en déclin dont le peuplement déjà limité est en diminution. Il s'git de Afraegle paniculata, Antiaris toxicaria, Carissa edulis, Commiphora africana, Crateva adansonii, Pseudocedrela kotschyi, Securidaca longipedunculata, Leptadenia hastata, Tylophora camerunica et Vernonia cinerea.

- Des plantes en déclin qui étaient répandues précédemment et dont les populations diminuent à cause de la destruction des populations sauvages. Il s'agit de Polygala arenaria, Pterocarpus santalinoides, Morinda lucida, Uvaria chamae, Phyllantus amarus, Ficus thonningii, Pycnanthus angolensis, Portulaca meridiana, Justicia flava et Uraria picta. 
Tableau 1: Caractéristiques démographiques, climatiques et édaphiques des sites prospectés.

\begin{tabular}{|c|c|c|c|c|}
\hline $\begin{array}{l}\text { Communes } \\
\text { Variables }\end{array}$ & Abomey & Allada & Ouidah & Cotonou \\
\hline Département & Zou & Atlantique & Atlantique & Littoral \\
\hline Superficie & $142 \mathrm{Km}^{2}$ & $381 \mathrm{~km}^{2}$ & $364 \mathrm{~km}^{2}$ & $79 \mathrm{~km}^{2}$ \\
\hline $\begin{array}{l}\text { Population (habitants) } \\
\text { (INSAE 2002) }\end{array}$ & 78.341 habitants & 91.778 habitants & 76.551 habitants & 761.137 habitants \\
\hline Activités principales & $\begin{array}{l}\text { - Agriculture } \\
\text { - Elevage } \\
\text { - commerce }\end{array}$ & $\begin{array}{l}\text { - Agriculture } \\
\text { - Elevage } \\
\text { - Commerce }\end{array}$ & $\begin{array}{l}\text { - Agriculture } \\
\text { - Pêche }\end{array}$ & $\begin{array}{l}\text { - Commerce } \\
\text { - Pêche }\end{array}$ \\
\hline Climat & $\begin{array}{l}\text { Guinéen: } 2 \text { saisons } \\
\text { de pluie et } 2 \text { saisons } \\
\text { sèches }\end{array}$ & $\begin{array}{l}\text { Guinéen: } 2 \text { saisons de } \\
\text { pluie et } 2 \text { saisons } \\
\text { sèches }\end{array}$ & Guinéen: 2 saisons de pluie et 2 saisons sèches & $\begin{array}{l}\text { Equatorial : } 2 \text { saisons de pluie et de } 2 \\
\text { saisons sèches }\end{array}$ \\
\hline Pluviométrie (mm/an) & $1133 \mathrm{~mm}$ & 800 et $1000 \mathrm{~mm}$ & 950 et $1150 \mathrm{~mm}$ & 800 à 1200 mm \\
\hline Température $\left({ }^{\circ} \mathrm{C}\right)$ & 27,50 & 26,80 & 27 & 27 \\
\hline
\end{tabular}


Tableau 2 : Drogues et usages de quelques espèces significatives.

\begin{tabular}{|c|c|c|c|c|}
\hline Espèces & Usages & Drogues végétales & Nombre de maladies traitées & Zone \\
\hline Afraegle paniculata (Schum. and Thonn.) Engl. & Med & Fel, R, Fr, Ec & 4 & Sud / Centre \\
\hline Aneilema lanceolatum Benth. & Sp, Med & Fel, fr, Ec; R & 5 & Sud \\
\hline Anogeissus leiocarpa (DC.) Guill. and Perr. & Med & Fel, R, tige, Ec & 11 & Sud \\
\hline Antiaris toxicaria Lesch. subsp. welwitschii (Engler) C.C. Berg & Sp, Med & Ec, sève, Fel R & 10 & Sud \\
\hline Boerhavia diffusa $\mathrm{L}$. & Med, Al & Fel, fr, Ec, graine; $\mathrm{R}$ & 9 & Sud / Centre \\
\hline Carissa edulis (Forssk.) Vahl & Med, Al & $\mathrm{R}, \mathrm{Fel}, \mathrm{Ec}$ & 15 & Sud / Centre \\
\hline Clausena anisata (Willd.) Benth. & Sp, Med & $\mathrm{R} ; \mathrm{Fel}$ & 7 & Sud \\
\hline Clitoria ternatea $\mathrm{L}$. & $\mathrm{Sp}$ & Fel & 1 & Sud \\
\hline Commiphora africana (A. Rich.) Engl. & Med, Sp & Fel & 4 & Sud / Centre \\
\hline Crateva adansonii DC. & Med & Fel, R & 9 & Sud / Centre \\
\hline Detarium senegalense J.F. Gmel. & Med & $\mathrm{R}$ & 1 & Sud / Centre \\
\hline Dichrostachys cinerea (L.) Wight and Arn. & Med & Fel & 9 & Sud \\
\hline Euphorbia convolvuloides Hochst. ex Benth. & Med & Fel, Sève, R & 3 & Sud / Centre \\
\hline Leptadenia hastata (Pers.) Decne & Med & Tige & 1 & Sud \\
\hline Maranthes kerstingii (Engl.) Prance & Med & Ec, Fel, R, tige & 4 & Sud \\
\hline Millettia thonningii (Schum. and Thonn.) Bak. & Med & Fel, tige & 3 & Sud \\
\hline Morinda lucida Benth. & Med & Fel, R & 7 & Sud \\
\hline
\end{tabular}


J. DJEGO et al. / Int. J. Biol. Chem. Sci. 5(4): 1432-1447, 2011

Paullinia pinnata $\mathrm{L}$.

Pavetta crassipes K. Schum.

Pericopsis laxiflora (Benth. ex Bak.) van Meeuwen

Polygala arenaria Willd.

Prosopis africana (Guill. and Perr.) Taub.

Pseudocedrala kotschyi (Schweinf.) Harms

Pterocarpus santalinoides DC.

Schwenckia americana $\mathrm{L}$.

Securidaca longipedunculata Fres.

Tephrosia vogelii Hook. f.

Vernonia amygdalina Del.

Vernonia cinerea (L.) Less.

\section{Med}

Med, Sp

Med

Med

Med

Med

Al, Med

Med

Med

Med

Al, Sp

Med, Sp
Fel, R

Fel, R, tige, Ec

Fel

$\mathrm{Fel}$

Fel, fr, R, Ec, Tige

tige, Ec, $\mathrm{R}$

Fr, Fel, Ec

Fel

Ec, $\mathrm{R}$

Fel

Fel

Fel, tige
Sud / Centre

Sud

Sud / Centre

Sud

Sud / Centre

Sud

Sud / Centre

Sud

Nord

Sud

Sud / Centre

Sud / Centre

Légende : $\mathrm{Al}=$ Alimentaire $;$ Med $=$ Médicinal $; \mathrm{Sp}=$ Spirituel $; \mathrm{Fel}=$ Feuille $; \mathrm{Fl}=$ Fleur $; \mathrm{Fr}=$ Fruit $; \mathrm{R}=$ Racine $; \mathrm{Ec}=$ Ecorce 


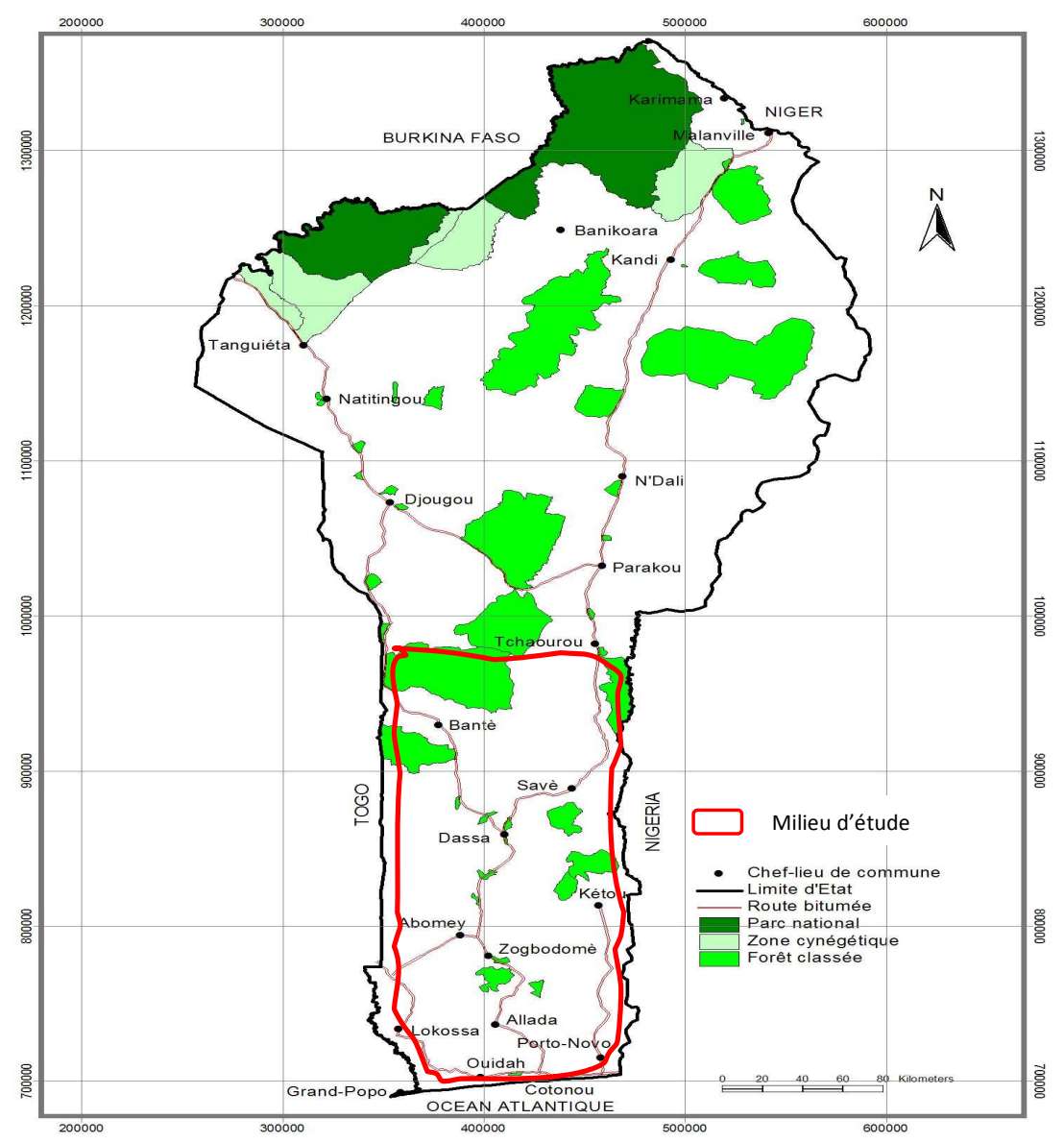

Figure 1 : Situation du milieu d'étude.

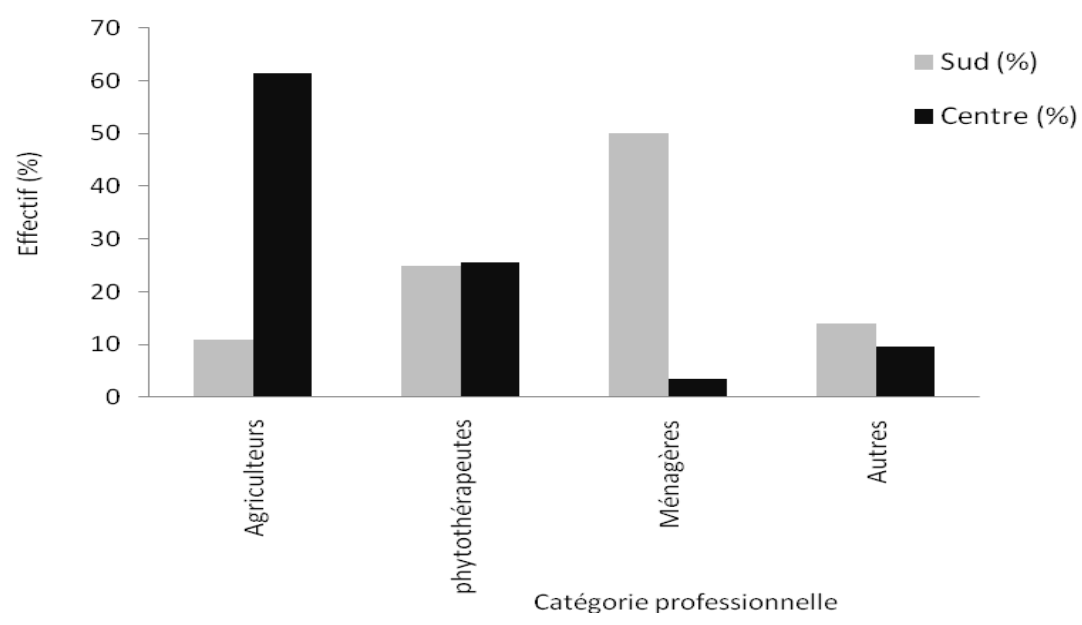

Figure 2 : Répartition de la population d'étude suivant les catégories professionnelles au Sud et au Centre Bénin. 


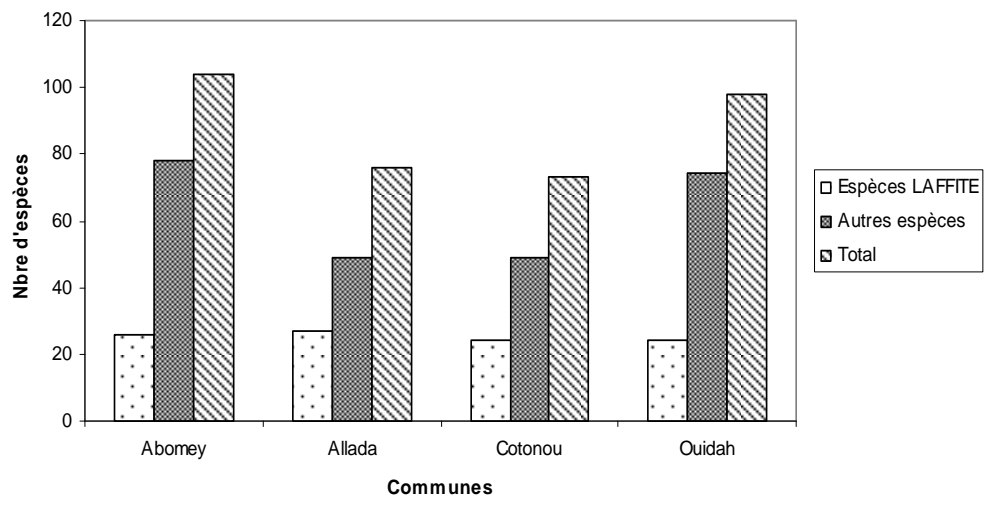

Figure 3 : Répartition du nombre d'espèces recensées par commune.

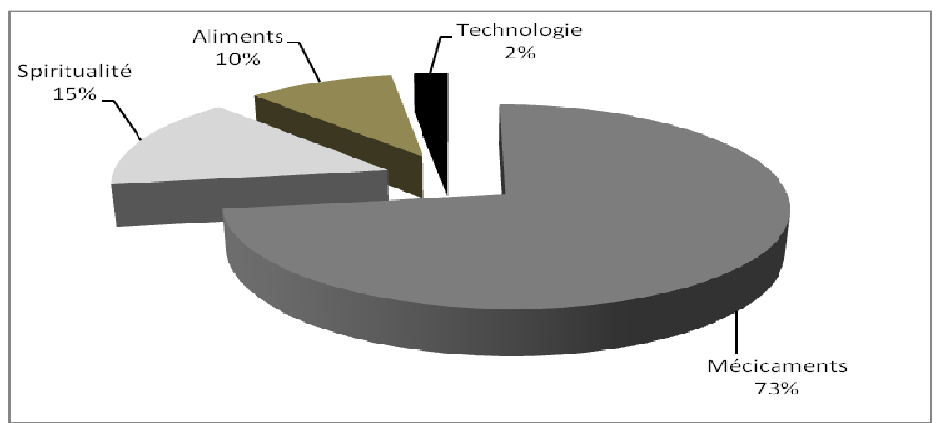

Figure 4 : Différentes utilisations des plantes médicinales par ordre d'importance au Sud et au Centre du Bénin.

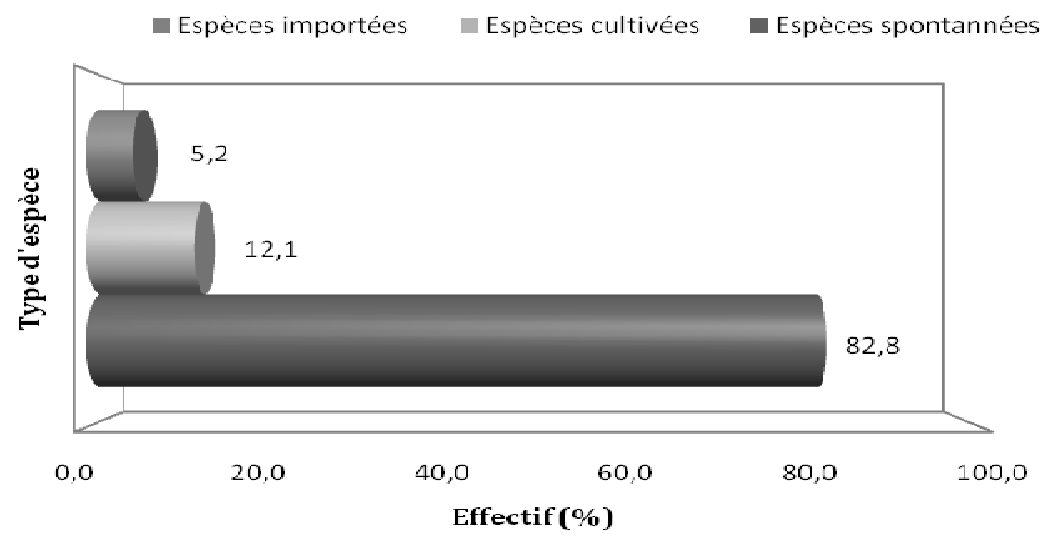

Figure 5 : Fréquence d'utilisation des plantes utiles selon leur provenance. 


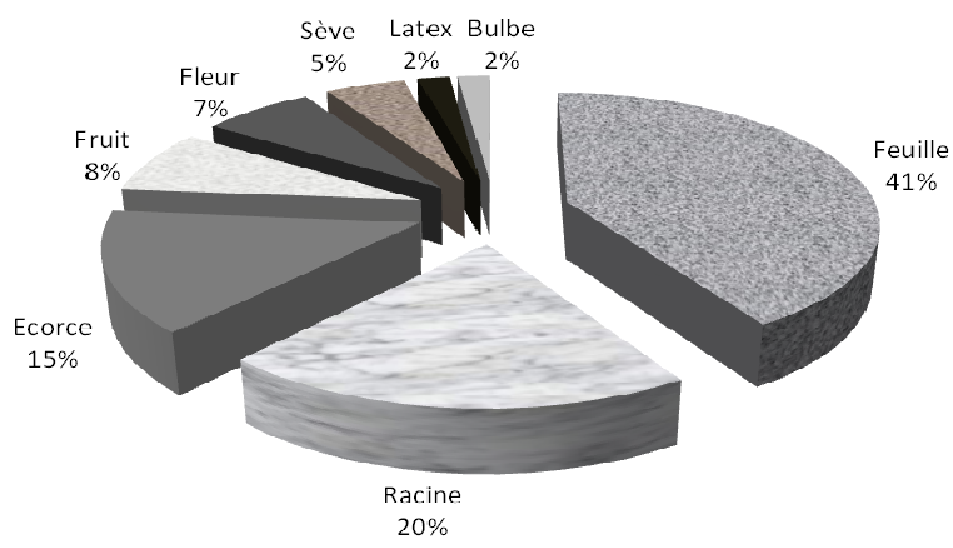

Figure 6 : Fréquence d'utilisation des drogues végétales au Sud et au Centre Bénin.

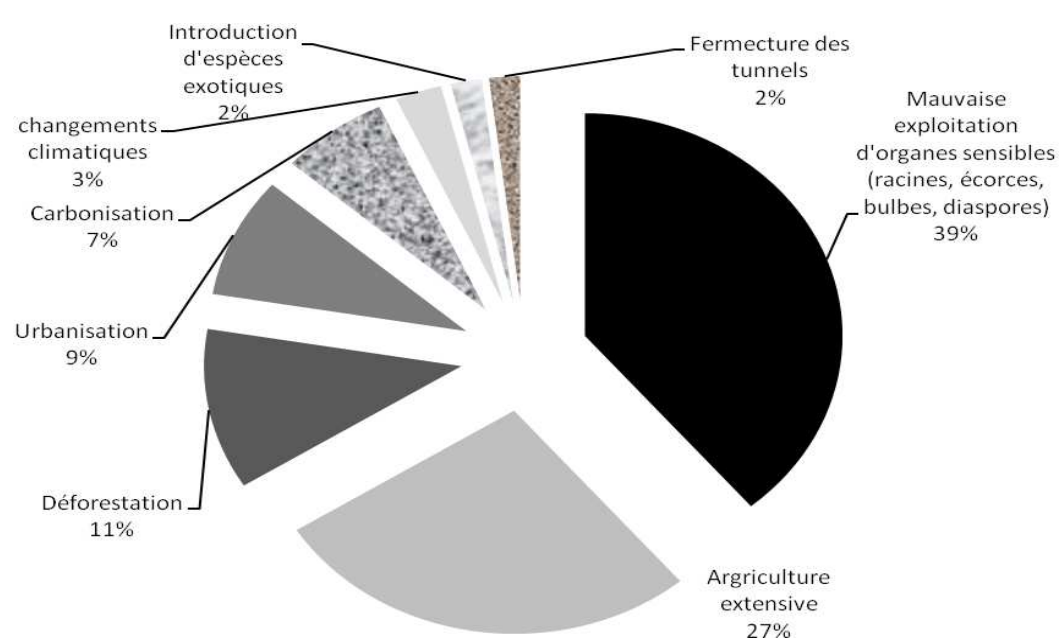

Figure 7: Causes de la vulnérabilité des espèces.

\section{DISCUSSION}

Impact des activités agricoles sur la vulnérabilité de la phytodiversité

Les aires de distribution des espèces végétales et plus particulièrement celles ayant des vertus thérapeutiques s'amenuisent. Les pratiques agricoles dévastatrices comme l'agriculture itinérante sur brûlis demeure la pratique culturale la plus dominante au Bénin (Adégbola et al., 2002; Goussanou et al., 2011) entraînant inévitablement l'utilisation d'espace très important. Ce qui est confirmé par Floquet and Mongbo (1998) qui affirment que l'agriculture provoque à court ou moyen terme une dégradation des terres et des écosystèmes. L'installation d'un champ passe par le défrichage et l'élimination des espèces compétitives des cultures. Celles qui sont épargnées dès le début de l'installation du champ finissent toujours par être éliminées du fait de l'isolement ou de l'exploitation. Seules, les espèces comme Parkia biglobosa, Vitellaria paradoxa et Securidaca longepedunculata fournissant peu d'ombrage sont parfois viables dans les champs et ceci surtout dans le Centre du pays. Les espèces comme Milletia thonningii, Afraegle paniculata, Premna hispida, Detarium 
senegalensis, Tephrosia vogelii, etc., sont menacées de disparition du fait de l'extension des surfaces agricoles suite à un accroissement des populations et une demande plus accrue des terres cultivables. L'exploitation incontrôlée de certaines espèces (Afraegle paniculata, Detarium senegalense, Tephrosia vogelii, etc.) ainsi que le mode de prélèvement des organes sensibles (racines, fruits, graines, écorce) sont aussi des causes de vulnérabilité des espèces. De pareils résultats sont en accord avec ceux obtenus par Sinsin et al. (2009) qui démontrent que les ressources végétales sont soumises à de fortes pressions humaines (agriculture, transhumance, émondage, feux de brousse, collecte des PFNL et occupation aux fins d'habitation) qui à divers degrés influencent négativement la conservation durable des forêts. Autres raisons évoquées par les enquêtés dans la régression des aires de distribution des plantes médicinales surtout au Sud Bénin, sont l'urbanisation, les changements climatiques et l'introduction d'espèces exotiques. La principale raison évoquée par les tradithérapeutes, les vendeuses ou les agriculteurs est l'urbanisation. Cette urbanisation étant perçue comme l'occupation des espaces par les infrastructures (routes, bâtiments, etc.), réduisant du coût les espaces occupés par les espèces (Kateb, 2004).

En ce qui concerne l'introduction d'espèces exotiques, ce facteur est essentiellement évoqué par rapport aux fruitiers où $10 \%$ des enquêtés ont affirmé que l'introduction de Tectona grandis par exemple a réduit considérablement la production des fruitiers comme celle de Pterocarpus santalinoides. L'introduction d'essences exotiques étant déjà été signalée comme nuisible au développement des espèces endogènes du milieu (Djègo and Sinsin, 2006).

\section{Impact des activités socio-économiques sur la vulnérabilité de la phytodiversité}

La valorisation des espèces passe par les usages qu'en font les populations. Les ligneux sont utilisés à diverses fins : bois de service, bois énergie, charbonnage, exploitation commerciale des racines et écorces des espèces médicinales très recherchées. Les espèces recensées sont souvent commercialisées au niveau des marchés. Les principales régions qui pourvoient ces marchés en plantes médicinales sont : Savalou, Dassa, Abomey et Covè. Dans les marchés, la quantité de racines et écorces vendues à 100 Francs CFA varie entre $100.0 \mathrm{~g}$ et $300.0 \mathrm{~g}$ pour la plupart des espèces sauf les racines de Carissa edulis dont $10.0 \mathrm{~g}$ sont vendues à 100 Francs CFA. Concernant les feuilles, la botte de $5.0 \mathrm{~g}$ est vendue à 25 Francs CFA. En moyenne la recette mensuelle est de $24.000 \mathrm{~F}$ CFA soit 1.000 F CFA par jour dans les communes d'Abomey, de Ouidah et d'Allada. Ce qui correspond à 240 bottes de racines par mois, soit $24.0 \mathrm{Kg}$ de racines vendus par mois; tandis qu'à Cotonou, 600 bottes de racines ou 60.0 Kg sont vendues mensuellement. Cet écart peut s'expliquer par le fait qu'à Cotonou la forte démographie et le faible pouvoir d'achat amènent la majorité des populations à recourir aux plantes médicinales. Les recettes journalières de ces communes (Abomey, Ouidah et Allada) confirment celles trouvées par Bonou (2008) et Toyi (2005) dans le Nord du Bénin. Cette commercialisation induit un prélèvement abusif des espèces sans aucune action de conservation. Ceci constitue la principale menace évoquée par les populations sur les plantes médicinales. Les raisons telles que la fermeture des tunnels, l'agriculture, la non culture de certaines espèces et autres causes (toxicité des graines, rituels destructeurs) sont également évoqués. Il est à noter que c'est uniquement à Abomey qu'il a été évoqué comme menace, la fermeture des tunnels (creusés pour servir de refuge aux 
populations durant les guerres ancestrales). Ces tunnels constituent de véritables réserves de ressources végétales et animales qui sont actuellement détruites à cause de l'urbanisation. Un autre cas évoqué dans la commune d'Abomey est l'abattage systématique de certaines espèces comme Cassia tora après certains rituels par les tradipraticiens. Bien que ce soit des pratiques rares, il n'en demeure pas moins qu'elles constituent une menace grave pour l'espèce. Dans la commune d'Allada, ce sont surtout les fruitiers (Spondias monbin, Uvaria chamae, etc.) qui ont été évoqués comme espèces menacées. A Ouidah, c'est plutôt Imperata cylindrica qui a été reconnu vulnérable, car il est systématiquement détruit des champs du fait de son caractère invasif; de plus, son rhizome est utilisé contre l'hypertension artérielle; le paludisme et l'ictère. Dans le Centre du pays, certaines espèces (Tephrosia vogelii et Premna hispida) sont très peu connues des populations du fait de leur très forte réduction dans le milieu.

En somme, les menaces qui pèsent sur les espèces sont réelles et préoccupantes (Figure 7). Elles sont liées principalement aux mauvaises exploitations d'organes sensibles (39\%), à l'agriculture extensive (27\%), à la déforestation (11\%) et à l'urbanisation $(9 \%)$. Elles sont dues aux prélèvements intenses, aux mutilations, à la déforestation incontrôlée ou mal gérée, aux pratiques agricoles dévastatrices (Adomou et al., 2007 ; Deleke et al., 2009 ; Delvaux et al., 2009).

Vue toutes ces pressions qui pèsent sur les plantes médicinales, quelles sont alors les mesures de conservation développées par la population pour assurer leur pérennité ?

\section{Mesure de protection}

La principale mesure de protection évoquée par les enquêtés est la mise en place de jardin de proximité ou jardin de case. 58\% des enquêtés (tradithérapeutes et les agriculteurs surtout) ont affirmé disposer de jardin qu'ils ont aménagé pour cultiver certaines espèces rares ou couramment utilisées. La commune d'Allada a plus de jardins (87\% des enquêtés). Viennent ensuite Abomey (60\%); Ouidah $(53 \%)$ et enfin Cotonou (31\%). Dans la commune d'Allada, au-delà des jardins de case, il a été observé dans l'arrondissement de Hinvi, un jardin botanique bien aménagé et clôturé. Ce jardin a été mis en place pour sauvegarder certaines plantes menacées telles que: Afraegle paniculata, Ficus exasperata; Milicia excelsa, Terminalia superba, Tamarindus indica, Cassia sieberiana, Tetrapleura tetraptera, Annona senegalensis, Khaya senegalensis, Zanthoxylum zanthoxyloides, etc. Les différentes espèces cultivées dans les maisons sont: Ocimum basilicum, Cymbopogon citratus, Ocimum gratissimum, Vernonia amygdalina, Carica papaya, etc. Les espèces rares cultivées dans les jardins sont: Pycnanthus angolensis, Uraria picta, Gossypium arboreum L., etc. Dans la commune d'Abomey, il a été également noté une autre forme de mise en place de jardin qui se fait comme un parterre où les espèces médicinales sont mélangées avec celles ornementales pour embellir les maisons et leur devanture.

Pour éviter la disparition d'espèces, la substitution d'organes sensibles (utilisation des feuilles par exemple à la place des racines) peut être envisagée. D'autres stratégies de prélèvement d'organes se pratiquent à savoir: le prélèvement des racines fasciculées à la place de la racine pivotante. En ce qui concerne l'écorçage, il est préférable qu'il se réalise d'un côté et non tout autour afin de favoriser la reconstitution de la partie manquante. De pareils constats ont été faits par Delvaux (2009) dans la forêt classée des Monts Kouffé.

Dans le centre du Bénin, outre Vernonia amygdalina (souvent cultivé par les femmes), Commiphora africana, et Crateva adansonii (d'usages courants) plantées dans 
les jardins de case, les populations préfèrent récolter les espèces dans la nature, même si elles sont dans des biotopes assez éloignés des habitations. Ainsi, la culture des plantes médicinales et la réglementation de la récolte des plantes spontanées pourraient réduire la pression sur les espèces végétales médicinales les plus utilisées en pharmacopée traditionnelle. Lorsqu'il s'agit de plantes rares, menacées d'extinction ou surexploitées en vue de leur commercialisation, la culture est la seule façon d'obtenir les quantités végétales nécessaires sans compromettre davantage la survie de ces espèces (OMS, UICN, WWF, 1993). Certes, il existe au niveau communal des jardins botaniques pour la conservation des plantes médicinales et le développement de la médicine traditionnelle. Mais au niveau village, rien n'est fait pour protéger les espèces menacées. La conception traditionaliste de la gestion des ressources naturelles est encore très présente dans les esprits des enquêtés qui supposent que la ressource est inépuisable bien qu'ils reconnaissent que des espèces disparaissent et que d'autres sont menacées de disparition. Il apparaît indispensable d'organiser des séances d'Information - d'Education - Communication pour éveiller les consciences sur la gestion durable des ressources naturelles et la nécessité de la conservation des espèces menacées de disparition.

\section{Conclusion}

La plupart des espèces recensées par Laffite sont présentes et sont exploitées dans le Sud et le Centre du Bénin (Abomey, Allada, Cotonou, Ouidah, Djidja, Glazoué, Dassa, Savalou et Bantè). Ces différentes espèces sont utilisées en médecine traditionnelle, dans l'alimentation, la spiritualité et la technologie. Elles font aussi objet de commercialisation et procurent des ressources financières aux populations. Du fait de leur utilité, les espèces recensées par Laffite sont très sollicitées et certaines se raréfient. En dehors des espèces recensées par Laffite, d'autres ont été identifiées et sont sujettes à de fortes exploitations. Mais, de plus en plus, certaines franges de la population ont commencé par prendre des dispositions en vue de leur conservation à travers la mise en place des jardins de case dans le Sud du Bénin. Dans le Centre, bien qu'il existe des mesures de conservation des ressources à travers les jardins botaniques au niveau communal, aucune stratégie de conservation pour contourner les menaces qui pèsent sur les espèces n'existe au niveau village. Ils utilisent les organes dont ils ont besoin car leur suivie en dépend; l'espèce peut mourir pourvu que l'homme soit sauvé.

Pour une réelle contribution à la pérennisation des espèces médicinales, il va falloir :

Organiser des séances de sensibilisation au sein des acteurs principaux qui interviennent dans le domaine des plantes médicinales; il s'agit notamment des tradithérapeutes et des vendeuses de plantes médicinales ;

- Poursuivre l'inventaire des plantes médicinales dans les quatre communes afin de constituer une liste plus exhaustive des principales espèces utilisées.

- Réaliser une base de données sur toutes les espèces médicinales ainsi que les différentes menaces qui pèsent sur ces espèces afin de capitaliser les informations pour une gestion plus efficace.

\section{REMERCIEMENTS}

Nous remercions, le projet Sud Expert Plantes qui a financé cette étude et son coordonnateur, le Docteur Mathieu Gueye de l'Institut Fondamental d'Afrique Noire (IFAN) de Dakar (Sénégal).

\section{REFERENCES}

Adégbola YP, Sodjinou E, Houssou N, Singbo AG. 2002. Etude financière et socioéconomique des techniques de gestion de 
la fertilité des sols au Sud-Bénin. INRAB, Prog. Anal. De la Polit. Agri. Porto-Novo. Bénin, P. 67.

Adjanohoun E, de Souza S, Eyog Matig O, Sinsin, B. 1999. Programme de ressources génétiques forestières en Afrique au sud du Sahara (programme SAFORGEN). Réseau Espèces Ligneuses Médicinales" Compte rendu de la première réunion du Réseau. IITA Cotonou, Bénin.

Adomou AC, Akoegninou A, Sinsin B, Defoucault B, Van der Maesen LGJ. 2007. Biogeographical analysis of the vegetation in Benin. Acta. Bot. Gall., 154: 221-233.

Bonou A. 2008. Estimation de la valeur économique des produits forestiers non ligneux (PFLN) d'origine végétale dans le village de Sampéto (commune de Banikoara). Mémoire de DEA, Faculté des Sciences Agronomiques, Université d'Abomey-Calavi, Bénin.

Deleke Koko IKE, Djègo J, HounzangbeAdote MS, Sinsin B. 2009. Etude ethnobotanique des plantes galactogènes et emménagogues utilisées dans les terroirs riverains à la Zone Cynégétique de la Pendjari. Int. J. Biol. Chem. Sci., 3(6): 1226-1237.

Delvaux C. 2009. Responses to bark harvesting of medicinal tree species from Forêt Classée des Monts Kouffé, Benin. $\mathrm{PhD}$ Thesis, Université de Ghent, Belgique, P.155.

Delvaux C, Sinsin B, Darchambeau F, Van Damme P. 2009. Recovery from bark harvesting of 12 medicinal tree species in Benin, West Africa. J. Appl. Ecol., 46: 703-712.

Delvaux C, Sinsin B, Van Damme P, Beeckman H, Van Damme P. 2010. Wound reaction after bark harvesting: microscopic and macroscopic phenomena in ten medicinal tree species (Benin). Trees, 24(5): 941-951.
Djègo JG. 2006. Phytosociologie de la végétation de sous-bois et impact écologique des plantations forestières sur la diversité floristique au sud et au centre Bénin. Thèse de Doctorat, Université d'Abomey-Calavi, Bénin, P. 359.

Djègo J, Sinsin B. 2006. Impact des espèces exotiques plantées sur la diversité des phytocénoses de leur sous-bois. Syst. Geogr. Pl., 76: $191-209$.

Djègo J, Oumorou M. 2009. Phytosociologie de sous-bois et impact des plantations forestières sur la diversité floristique dans la forêt classée de la Lama. Annales des Sciences Agronomiques du Bénin, 12(1): 35-54.

Floquet A, Mongbo RL. 1998. Des Paysans en Mal d'Alternatives. Dégradation des Terres, Restructuration de l'Espace Agraire et Urbanisation au bas Bénin. Margraf Verlag: Weikersheim, P. 190.

FAO. 1999. The major significance of «minor» forest product: local people's user and values of forests in the West African humid forest zone. Community Forest Note1, Rome.

Goussanou C, Tenté B, Djègo J, Agbani P, Sinsin B. 2011. Inventaire, caractérisation et mode de gestion de quelques produits forestiers non ligneux du Bassin versant de la Donga. Ann. Sc.Agro., 14(1): 77-99.

Harrison P. 1991. Une Afrique Verte. CTA: Wageningen, Pays-Bas; P. 448.

Kateb K. 2004. Place et rôle des petites et moyennes agglomérations dans les nouvelles dynamiques urbaines en Algérie. Réseau des chercheurs de l'AUF. INED, France, P. 16.

OMS, UICN, WWF. 1993. Principes directeurs pour la conservation des plantes médicinales, Gland, Suisse, P. 35.

Ros-tonen M. 1999. NTFP research in Tropenbos program. Tropenbos Newsletter, 19: 3-4.

Sinsin B, Djègo J, Adomou A, Houéssou L. 2009. Etude ethnobotanique des forêts 
classées de Goungoun, de Sota et de la rôneraie de Goroubi au Bénin. Rapport d'étude Cerget-Ong/PGFTR, Bénin, P. 91.

Sokpon N, Lejoly J. 1996. Les plantes alimentaires d'une forêt dense caducifoliée, Pobè au sud du Bénin. In L'Alimentation en Forêt Tropicale: Interactions Bioculturelles et Perspectives de Développement, Hladik CM, Hladik A, Linares OF, Koppert GJA, Froment A (eds). Editions UNESCO: Paris.

Sokpon N, Ago EE. 2001. Sacralisation et niveau de maturation des forêts denses semi-décidues du Plateau Adja au SudOuest du Bénin. J. Rech. Univ. Lomé, 5(2): 319-331.

Tabuti JRS, Lye KA, Dhillion SS. 2003. Traditional herbal drugs of Bulamogi,
Uganda: plants, use and administration. $J$. Ethnopharmacology, 88: 19-44.

Toyi MS. 2005. Les principales espèces végétales utilisées dans la médecine traditionnelle dans la commune de Péhunco: mode d'exploitation, abondance, et dynamique de régénération. Mém. CPU/UAC, Bénin, P. 140.

Yessoufou K. 2005. Recherches ethnobotaniques et écologiques sur deux espèces fruitières dans le Département du Plateau, Sud-Bénin: Irvingia gabonensis (Aubry-Lecomte ex O'Rorke) Baill. et Blighia sapida K. Konig. Th. DESS.AGRN.FSA.UAC, Bénin, P. 67. 